\title{
Organic and Inorganic Passivation of p-type SnO Thin-Film Transistors with Different Active Layer Thickness
}

DOI:

10.1088/1361-6641/aac3c4

\section{Document Version}

Accepted author manuscript

Link to publication record in Manchester Research Explorer

\section{Citation for published version (APA):}

Qu, Y., Yang, J., Li, Y., Zhang, J., Wang, Q., Song, A., \& Xin, Q. (2018). Organic and Inorganic Passivation of ptype SnO Thin-Film Transistors with Different Active Layer Thickness. Semiconductor Science and Technology, 33(7), [075001]. https://doi.org/10.1088/1361-6641/aac3c4

\section{Published in:}

Semiconductor Science and Technology

\section{Citing this paper}

Please note that where the full-text provided on Manchester Research Explorer is the Author Accepted Manuscript or Proof version this may differ from the final Published version. If citing, it is advised that you check and use the publisher's definitive version.

\section{General rights}

Copyright and moral rights for the publications made accessible in the Research Explorer are retained by the authors and/or other copyright owners and it is a condition of accessing publications that users recognise and abide by the legal requirements associated with these rights.

\section{Takedown policy}

If you believe that this document breaches copyright please refer to the University of Manchester's Takedown Procedures [http://man.ac.uk/04Y6Bo] or contact uml.scholarlycommunications@manchester.ac.uk providing relevant details, so we can investigate your claim.

\section{OPEN ACCESS}




\title{
Organic and Inorganic Passivation of p-type SnO Thin-Film Transistors with Different Active Layer Thicknesses Yunxiu Qu, ${ }^{1,2}$ Jia Yang, ${ }^{1,2}$ Yunpeng Li, ${ }^{1,2}$ Jiawei Zhang, ${ }^{3}$ Qingpu Wang, ${ }^{1}$ Aimin Song, ${ }^{1,2,3}$ and Qian Xin ${ }^{1,2 a)}$ \\ ${ }^{1}$ Center of Nanoelectronics, State Key Laboratory of Crystal Materials, and School of Microelectronics, Shandong \\ University, Jinan 250100, China \\ ${ }^{2}$ Suzhou Institute of Shandong University, Suzhou 215123, China \\ ${ }^{3}$ School of Electrical and Electronic Engineering, University of Manchester, Manchester M13 9PL, United \\ Kingdom
}

\begin{abstract}
Bottom gated thin-film transistors (TFTs) with various sputtered $\mathrm{SnO}$ active layer thicknesses ranging from 10 to $30 \mathrm{~nm}$ and different passivation layers have been investigated. The device with $20 \mathrm{~nm} \mathrm{SnO}$ showed the highest on/off ratio of $1.7 \times 10^{4}$ and the smallest subthreshold swing of $8.43 \mathrm{~V} / \mathrm{dec}$, and the mobility $\left(0.76 \mathrm{~cm}^{2} / \mathrm{Vs}\right)$ was only slightly lower than in TFTs with a thicker SnO layer. However, both the mobility and the on/off ratio of the $15 \mathrm{~nm}$ SnO TFT dropped significantly by one order of magnitude. This indicated a strong influence of the top surface on the carrier transport, and we thus applied an organic or an inorganic encapsulation material to passivate the top surface. In the $20 \mathrm{~nm}$ TFT, the on/off ratio was doubled after passivation. The performance of the $15 \mathrm{~nm}$ TFT was improved even more dramatically with the on/off ratio increased by one order of magnitude and the mobility increased also significantly. Our experiment shows that polymethyl methacrylate passivation is more effective to reduce the shallow trap states, and $\mathrm{Al}_{2} \mathrm{O}_{3}$ is more effective in reducing the deep traps in the $\mathrm{SnO}$ channel.
\end{abstract}

Keywords: $\mathrm{SnO}$, thin-flim transistors, top surface, passivation 


\section{Introduction}

Oxide semiconductors have recently received significant attention especially in the perspective of fast growing flexible and/or transparent electronics, such as flexible displays and wearable electronics, because of their low processing temperature compatible with plastic substrates, high carrier mobility $\left(\sim 1-100 \mathrm{~cm}^{2} \mathrm{~V}^{-1} \mathrm{~s}^{-1}\right)$, high optical transparency in visible light region, and low cost in comparison to amorphous silicon [1-3]. However, one major challenge for oxide semiconductors is that most of them are n-type, and only a very limited number of oxides show p-type conductivity. The reported thin-film transistors (TFTs) based on p-type oxides usually show lower mobilities and much lower on/off ratios than their n-type counterparts [4]. Such unbalanced development of $\mathrm{n}$ - and p-type oxides strongly hampers the implement of $\mathrm{p}-\mathrm{n}$ oxide junction based electronics and complementary metal oxide semiconductor integrated circuits with low-power consumption.

Among the reported p-type oxide semiconductors, $\mathrm{SnO}$ is considered as one of the most promising due to its native p-type conductivity, high mobility, good stability and reproducibility [4-10]. The valence band maximum of SnO is composed by the hybridization of $\mathrm{O} 2 p$ and delocalized spherical Sn $5 s$ orbitals, thus leading to a small hole effective mass and relatively high mobility [5,11]. The first-principle calculations suggested that the p-type conductivity of $\mathrm{SnO}$ could be enhanced by tin vacancies which have lower formation energy comparing with other defects, for example, oxygen vacancies [12]. The two main problems hindering the industrialization progress of p-type SnO TFTs are the low on/off ratio and large subthreshold swing (SS). The gap state density in a SnO TFT channel is typically quite high, and such a high density usually leads to a large $S S$ value and high off current because it is difficult to raise the Fermi level by gate voltage [13]. Recent studies showed that SnO thickness may play an important role on such gap state density [10,14-16]. The gap state density in SnO TFTs can be reduced and ambipolar behavior can be achieved by selecting the appropriate channel thickness [14-16]. It is known that the gap state density in oxide semiconductor TFTs can be affected by the ambient moisture and oxygen adsorption on the top channel surface [17-19]. Moisture adsorbents can donate electrons and act as hole traps, and oxygen adsorbents can capture electrons [20]. Recently, it was found that top surface passivation on bottom-gated SnO TFTs could suppress the surface states and shield the channel layer from the ambient atmosphere [7,10,21]. SnO TFTs with SU-8 passivation showed much improved long-term durability and bias stress stability under ambient air compared to those unpassivated [21]. It has been demonstrated that $\mathrm{Al}_{2} \mathrm{O}_{3}$ encapsulation on SnO TFTs not only improved electrical performance but also enabled ambipolar behavior by reducing the gap states [7].

In this work, we have systematically explored the influence of different active layer thicknesses and passivation layers on the performance of SnO TFTs. The effect of SnO layer thickness on the hole mobility is found not remarkable for TFTs with SnO thickness in a range of $20 \sim 30 \mathrm{~nm}$, but the mobility in the $15 \mathrm{~nm}$ device drops by one order of magnitude. In order to 
study the effect of different passivation layers on SnO TFTs with different active layer thicknesses, organic polymethyl methacrylate (PMMA) and inorganic $\mathrm{Al}_{2} \mathrm{O}_{3}$ are used to encapsulate the devices. Bias stress stabilities in SnO TFTs with and without encapsulation are also studied.

\section{Experiments}

The SnO TFTs were fabricated on thermally-oxidized $\mathrm{SiO}_{2}(\sim 100 \mathrm{~nm}) / \mathrm{P}^{+}-\mathrm{Si}$ substrates. SnO thin-films were deposited at room temperature by radio-frequency magnetron sputtering using a Sn target. The sputtering power, working pressure, and oxygen partial pressure $\left(\mathrm{O}_{2} /\left(\mathrm{O}_{2}+\mathrm{Ar}\right)\right)$ were $50 \mathrm{~W}, 3.5 \mathrm{mTorr}$, and $3.1 \%$, respectively. The as-deposited SnO films were annealed at $225^{\circ} \mathrm{C}$ in ambient air for 2 hours to achieve polycrystalline phase for high performance p-type conductivity. 50 -nm-Ti was deposited as the source and drain electrodes with a shadow mask by an electron-beam evaporator. The active channel length and width were 60 and $2000 \mu \mathrm{m}$, respectively. To study the effect of passivation, some of the SnO TFTs were passivated with either $\mathrm{Al}_{2} \mathrm{O}_{3}(50 \mathrm{~nm})$ or PMMA $(250 \mathrm{~nm})$ layer. The $\mathrm{Al}_{2} \mathrm{O}_{3}$ films were grown by atomic-layer deposition using trimethylaluminium and deionized water as the oxidizer and nitrogen as the carrier gas, at a chamber temperature of $150{ }^{\circ} \mathrm{C}$. The PMMA passivation layers were deposited by spincoating, and the PMMA passivated devices were annealed in air at 150 ${ }^{\circ} \mathrm{C}$ for 1 hour. The current-voltage characteristics of these TFTs were measured in dark using a source/measure unit (Aglient B2902A).

\section{Results and discussion}

Figures 1(a)-(b) illustrate the transfer and output characteristics of SnO TFTs with different channel thicknesses in a range of 10-30 nm. P-type behavior is obtained when the SnO thickness is 15, 20, 25 or $30 \mathrm{~nm}$. The output characteristics of the ptype TFTs exhibit clear pinch-off and saturated regions, and the typical one with 20-nm SnO is shown in Fig. 1(b). Electrical properties of all devices, including linear field effect mobility $\left(\mu_{\text {lin }}\right)$, threshold voltage $\left(V_{\mathrm{th}}\right), S S$, and on/off current ratio $\left(I_{\mathrm{on}} / I_{\mathrm{off}}\right)$, are summarized in Table 1. The device with a $20 \mathrm{~nm} \mathrm{SnO}$ active layer shows the best performance with the highest on/off ratio of $1.7 \times 10^{4}$ and the lowest $S S$ of $8.43 \mathrm{~V} / \mathrm{dec}$.
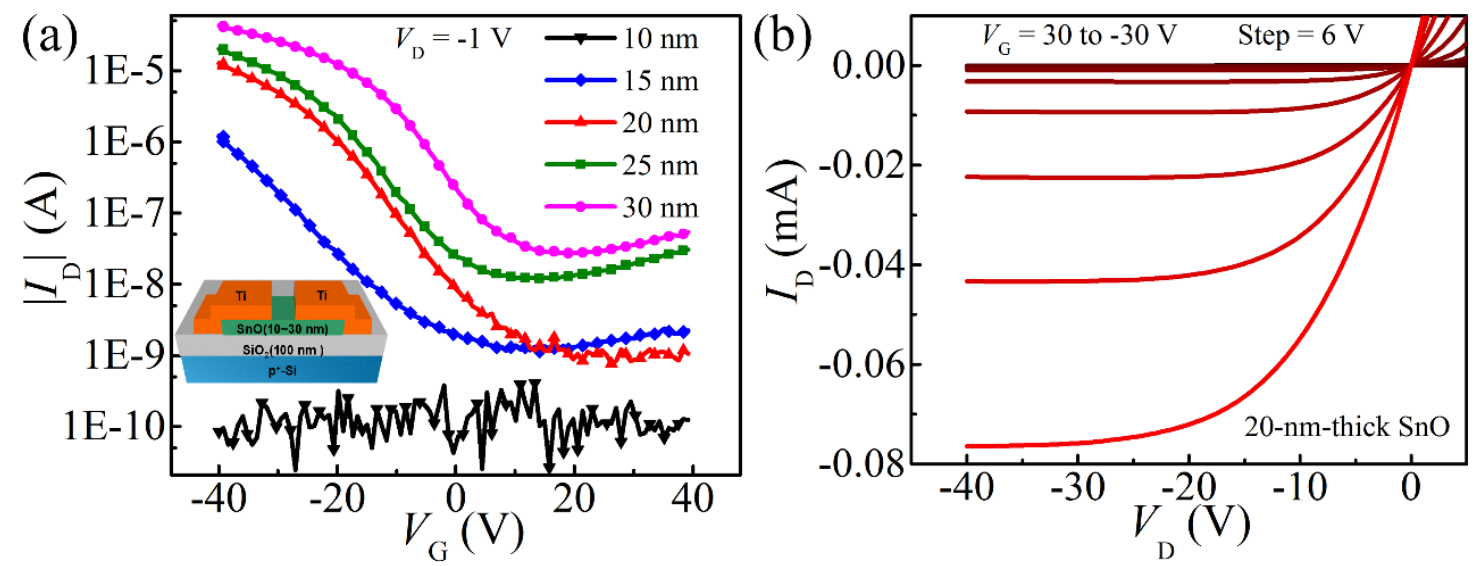
FIG. 1. (a) Transfer characteristics of TFTs with SnO of various thicknesses; (b) Output characteristics of TFT with a $20 \mathrm{~nm}$ SnO active layer.

TABLE 1. Performance parameters of TFTs with various SnO thicknesses.

\begin{tabular}{ccccc}
\hline Thickness $(\mathrm{nm})$ & $V_{\text {th }}(\mathrm{V})$ & $\mu_{\text {lin }}\left(\mathrm{cm}^{2} / \mathrm{Vs}\right)$ & $S S(\mathrm{~V} / \mathrm{dec})$ & $I_{\mathrm{on}} / I_{\text {off }}$ \\
\hline 10 & - & - & - & - \\
15 & -31.0 & 0.07 & 12.5 & $1.0 \times 10^{3}$ \\
20 & -26.5 & 0.76 & 8.43 & $1.7 \times 10^{4}$ \\
25 & -21.1 & 0.88 & 9.16 & $1.2 \times 10^{3}$ \\
30 & -13.3 & 1.41 & 9.55 & $1.5 \times 10^{3}$ \\
\hline
\end{tabular}

As the $\mathrm{SnO}$ thickness decreases, the TFT shows a clear decrease in on current, and the $V_{\text {th }}$ shifts towards negative direction. In the case of the $10 \mathrm{~nm} \mathrm{SnO}$, the channel current becomes very low, around $10^{-10} \mathrm{~A}$, and no gate modulation is observed. First of all, decreasing the channel thickness will mean fewer free carriers in the channel. The other key reason is the influence of the top channel surface. Moisture adsorbents and native tin dioxide on the top channel surface can act as electron donors which may complement the holes and act as hole traps [16,22]. The TFTs with thinner SnO channels have lower mobility, as shown in Table 1, indicating that there are a large number of shallow traps [23,24]. It's worth noting that the hole mobility of the TFT with $15 \mathrm{~nm} \mathrm{SnO}, 0.07 \mathrm{~cm}^{2} / \mathrm{Vs}$, is one order of magnitude lower than that of the TFT with $20 \mathrm{~nm} \mathrm{SnO} \mathrm{layer,} 0.76 \mathrm{~cm} / \mathrm{Vs}$. This indicates that the shallow traps on the top channel surface largely limits the hole transportation in very thin $\mathrm{SnO}$ channels $(\leq$ $15 \mathrm{~nm})$.

It is clearly seen that $S S$ decreases with $\mathrm{SnO}$ thickness decreases from 30 to $20 \mathrm{~nm}$. SS value is associated with the total trap density $\left(D_{\text {total }}\right)$ [25], consisting of the interface trap density $\left(D_{\text {it }}\right)$ at the $\mathrm{SnO} /$ gate insulator interface, the trap density of bulk SnO film $\left(D_{\mathrm{b}}\right)$ and the trap density of SnO top surface $\left(D_{\mathrm{top}}\right)$ :

$$
S S=\ln 10 \frac{k_{\mathrm{B}} T}{q}\left(1+\frac{q^{2} D_{\text {total }}}{C_{\mathrm{G}}}\right)
$$

where $q$ is the electron charge, $k_{\mathrm{B}}$ is the Boltzmann constant, $T$ is the absolute temperature, and $C_{\mathrm{G}}$ is the gate capacitance per unit area. As all the SnO layers with different thicknesses were deposited with the same processing parameters, a uniform bulk trap density $D_{\mathrm{b}}$ can be assumed, and $D_{\text {total }}$ can be expressed as,

$$
D_{\text {total }}=D_{\text {it }}+t D_{\mathrm{b}}+D_{\text {top }}
$$

where $t$ is the thickness of the SnO layer. For TFTs with SnO thickness from 30 to $20 \mathrm{~nm}$, the decrease of $S S$ can be explained 
by the reduction of $t$. It should be noticed that $S S$ of the TFT with $15 \mathrm{~nm}$ thick SnO layer was obviously larger than the TFTs with thicker SnO. This suggests that the top surface starts to become quite dominant as the SnO decreases to less than $\sim 15 \mathrm{~nm}$.

To suppress the influence of $D_{\text {top }}$, we have passivated the $\mathrm{SnO}$ top surface by $\mathrm{PMMA}$ or $\mathrm{Al}_{2} \mathrm{O}_{3}$. Figures 2 (a)-(b) and (c)(d) illustrate the transfer and output curves for the unpassivated, PMMA or $\mathrm{Al}_{2} \mathrm{O}_{3}$ passivated TFTs with $20 \mathrm{~nm} \mathrm{SnO}$ and $15 \mathrm{~nm}$ SnO, respectively. The results clearly show that the TFTs with passivation layers show improved performance in contrast to those unpassivated. In the $20 \mathrm{~nm}$ thick SnO TFTs, $S S$ reduces from 8.43 to 6.65 and $6.78 \mathrm{~V} /$ decade, and mobility increases from to 0.76 to 0.81 and $1.00 \mathrm{~cm}^{2} / \mathrm{Vs}$, after $\mathrm{Al}_{2} \mathrm{O}_{3}$ and PMMA passivation, respectively. A notable increase of on-current and on/off ratio is also observed. In the $15 \mathrm{~nm}$ thick SnO TFTs, the passivations are more effective as $S S$ reduces from 12.5 to 8.77 and $10.00 \mathrm{~V} / \mathrm{dec}$ ade, and mobility increases from 0.07 to 0.16 and $0.24 \mathrm{~cm}^{2} / \mathrm{Vs}$, after $\mathrm{Al}_{2} \mathrm{O}_{3}$ and PMMA passivation, respectively. Detailed device performance parameters are shown in Table 2. These results confirm that the passivation of top SnO surface can lead to an improvement of the TFT performance by attenuation of the ambient adsorption effect and reducing the trap density [10].

TABLE 2. Performance parameters of the TFTs with different SnO thicknesses, with and without passivation.

\begin{tabular}{cccccc}
\hline Thickness $(\mathrm{nm})$ & Passivation & $V_{\text {th }}(\mathrm{V})$ & $\mu_{\text {lin }}\left(\mathrm{cm}^{2} / \mathrm{Vs}\right)$ & $S S(\mathrm{~V} / \mathrm{dec})$ & $I_{\text {on }} / I_{\text {off }}$ \\
\hline \multirow{2}{*}{15} & without & -31.0 & 0.07 & 12.5 & $1.0 \times 10^{3}$ \\
& with $\mathrm{Al}_{2} \mathrm{O}_{3}$ & -22.9 & 0.16 & 8.77 & $1.0 \times 10^{4}$ \\
& with PMMA & -23.5 & 0.24 & 10.0 & $9.6 \times 10^{3}$ \\
\hline \multirow{2}{*}{20} & without & -26.5 & 0.76 & 8.43 & $1.7 \times 10^{4}$ \\
& with $\mathrm{Al}_{2} \mathrm{O}_{3}$ & -12.0 & 0.81 & 6.65 & $3.8 \times 10^{4}$ \\
& with PMMA & -9.10 & 1.00 & 6.78 & $3.3 \times 10^{4}$ \\
\hline
\end{tabular}



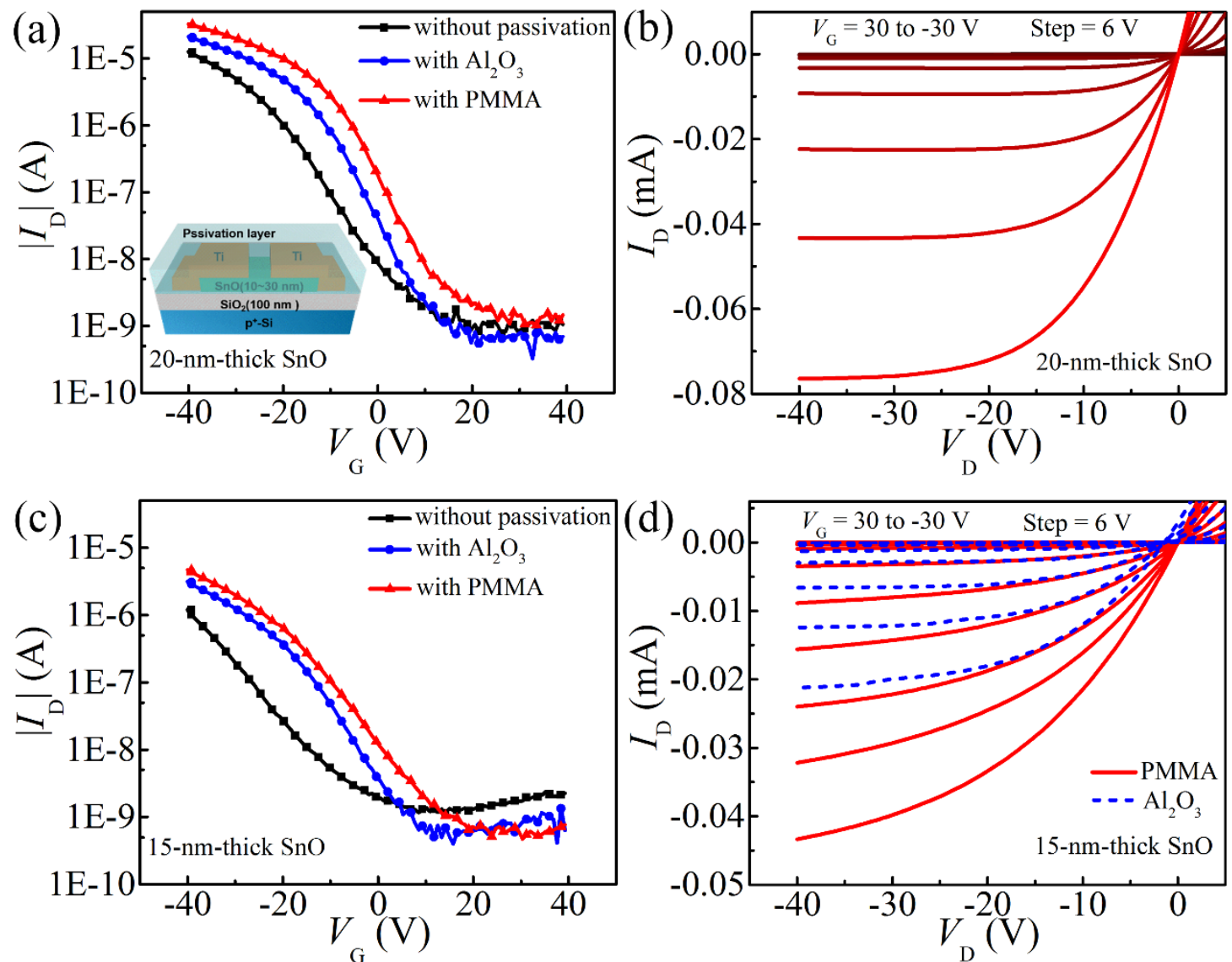

FIG. 2. Transfer (a) and Output (b) characteristics of 20-nm-thick SnO TFTs without and with passivation; Transfer (c) and Output (d) characteristics of 15 -nm-thick SnO TFTs without and with passivation.

In TFTs, the carrier mobility is related to the density of the tailing states near the conduction/valence band (shallow traps) and $S S$ is determined by the density of both deep and shallow traps $[14,23,24,26]$. It is interesting to note that the $\mathrm{Al}_{2} \mathrm{O}_{3}$ passivation leads to better on/off ratios and lower $S S$ values in both $15 \mathrm{~nm}$ and $20 \mathrm{~nm}$ SnO TFTs, whereas the PMMA passivation enables higher mobility. The experiment therefore suggests that the PMMA passivation is more effective than $\mathrm{Al}_{2} \mathrm{O}_{3}$ to reduce shallow trap states on the $\mathrm{SnO}$ top surface, while $\mathrm{Al}_{2} \mathrm{O}_{3}$ is more effective than PMMA in reducing the deep traps on the top surface of the $\mathrm{SnO}$ film. 

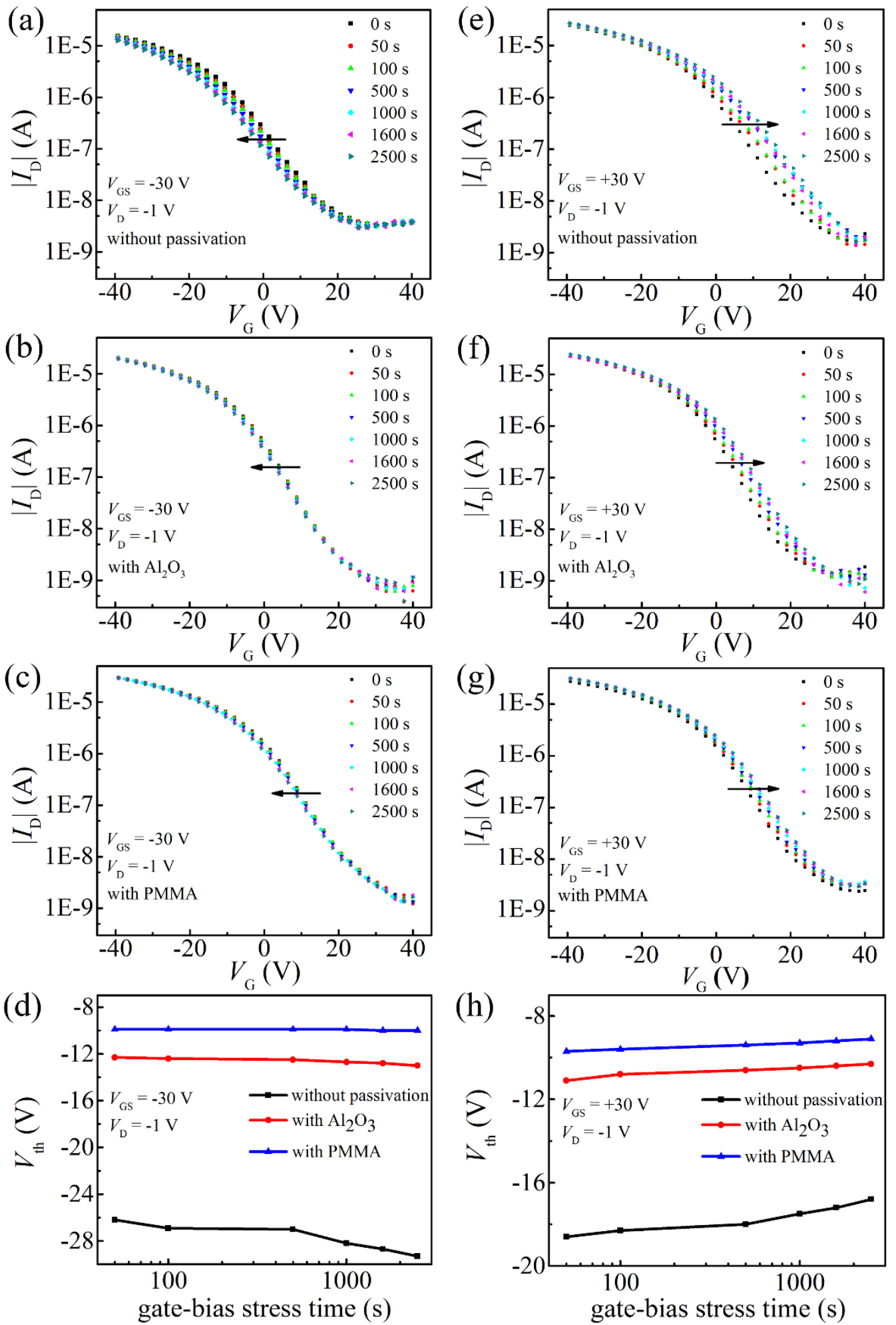

FIG.3. Time evolution of the transfer curves under a negative gate bias stress of $V_{\mathrm{GS}}=-30 \mathrm{~V}$ of $\mathrm{SnO}_{\mathrm{TFTs}}$ without passivation (a), with $\mathrm{Al}_{2} \mathrm{O}_{3}$ passivation (b), with PMMA passivation (c), and $V_{\text {th }}$ as a function of the negative gate-bias stress time (d).Time evolution of the transfer curves under a positive gate bias stress of $V_{\mathrm{GS}}=+30 \mathrm{~V}$ of SnO TFTs without passivation (e), with $\mathrm{Al}_{2} \mathrm{O}_{3}$ passivation (f), and with $\mathrm{PMMA}$ passivation (g), and $V_{\text {th }}$ as a function of the positive gate-bias stress time (h). 
Figures 3(a)-(d) show $V_{\text {th }}$ instability of SnO TFTs with or without passivation layer under -30 V negative gate bias stress with various stress time. The transfer characteristic is negatively shifted as the stress time increases, and $V_{\text {th }}$ shift is more pronounced for unpassivated TFTs. Because all the TFTs without or with passivation have the identical gate dielectric and SnO layer, the charge trapping at the channel-dielectric interface would be expected to be very similar. Thus the different $V_{\text {th }}$ shifts can be attributed to the ambient adsorption effect. For the unpassivated sample, when a negative gate bias was applied, the vertical electric field would enhance the adsorption of moisture on the top channel surface because water molecules can be positively charged by donating electrons [22]. Meanwhile, the applied negative gate bias can also enhance the desorption of oxygen molecules because they can be negatively charged by capturing electrons $[19,20]$.Thus, with the increase of negative bias stress time, more negative $V_{\text {th }}$ shift occurs due to the increased hole traps and decreased hole carrier density [20]. Such $V_{\text {th }}$ shift is reduced when $\mathrm{Al}_{2} \mathrm{O}_{3}$ or PMMA passivation reduces the adsorption of moisture and desorption of oxygen. Figures 3(e)(h) show the time evolution of the transfer curves and $V_{\text {th }}$ as a function of gate-bias stress time under a positive gate bias stress of $V_{\mathrm{GS}}=+30 \mathrm{~V}$. In all devices, transfer curves shift towards the positive direction as the stress time increases, which is due to the increased hole carrier density caused by the positive gate bias induced desorption of moisture and adsorption of oxygen. Similarly, the TFTs with $\mathrm{Al}_{2} \mathrm{O}_{3}$ or PMMA encapsulation also significantly improve the electrical stability by reducing the ambient adsorption effect.

\section{Conclusions}

In summary, we have studied the separate effects of active layer thickness and passivation on the performance of p-type SnO TFTs. For unpassivated TFTs with SnO thickness in range of $20 \sim 30 \mathrm{~nm}$, the field effect mobility, on current, and $S S$ are mainly limited by the amount of bulk film traps, and thus thinner $\mathrm{SnO}$ with less amount of bulk traps shows better performance. However, for unpassivated TFTs with $\mathrm{SnO}$ thickness $\leq 15 \mathrm{~nm}$, the performance is mainly limited by the top channel surface trap states caused by the ambient adsorption effect. $\mathrm{SnO}$ top surface passivation can effectively reduce the trap density on the top interface, and thus improve the TFT performance including mobility, on/off ratios, bias stability, and $S S$. $\mathrm{The}^{\mathrm{Al}_{2} \mathrm{O}_{3}}$ passivation is found to enable better on/off ratios and lower $S S$ values than the PMMA passivation. The PMMA passivation results in better mobility enhancement than the $\mathrm{Al}_{2} \mathrm{O}_{3}$ passivation. The results suggest that PMMA passivation is more effective to reduce the shallow trap states, and $\mathrm{Al}_{2} \mathrm{O}_{3}$ is more effective in reducing the deep traps in the $\mathrm{SnO}$ channel. By applying a $\mathrm{SnO}$ thickness of $20 \mathrm{~nm}$ and $\mathrm{Al}_{2} \mathrm{O}_{3}$ passivation, the optimized TFT achieves a high on/off ratio of $\sim 3.8 \times 10^{4}$, high mobility of 0.81 $\mathrm{cm}^{2} / \mathrm{Vs}$, and small $S S$ of $6.65 \mathrm{~V} / \mathrm{dec}$.

\section{Acknowledgments}


This work was supported by the National Key Research and Development Program of China (Grant No. 2016 YFA0301200 and 2016YFA0201800), the National Natural Science Foundation of China (Grant No. 11374185 and 11304180), Engineering and Physical Sciences Research Council (EPSRC) (Grant No. EP/N021258/1), China Postdoctoral Science Foundation funded project (2016M590634), the Key Research and Development Program of Shandong Province (2017GGX10111 and 2017GGX10121), the Natural Science Foundation of Jiangsu Province (BK20151255), Suzhou Planning Projects of Science and Technology (SYG201527 and SYG201616), and the Fundamental Research Fund of Shandong University (2016WLJH44).

\section{References}

[1] Fortunato E, Barquinha P and Martins R 2012 Oxide Semiconductor Thin-Film Transistors: A Review of Recent Advances Adv. Mater. 24 2945-86

[2] Nomura K, Ohta H, Takagi A, Kamiya T, Hirano M and Hosono H 2004 Room-Temperature Fabrication of Transparent Flexible Thin-Film Transistors Using Amorphous Oxide Semiconductors Nature 432 488-92

[3] Kim B, Cho H N, Choi W S, Kuk S H, Jang Y H, Yoo J S, Yoon S Y, Jun M, Hwang Y K and Han M K 2012 Highly Reliable Depletion-Mode a-IGZO TFT Gate Driver Circuits for High-Frequency Display Applications Under Light Illumination IEEE Electron Device Lett. 33 528-30

[4] Wang Z, Nayak P K, Caraveo-Frescas J A and Alshareef H N 2016 Recent Developments in p-Type Oxide Semiconductor Materials and Devices Adv. Mater. 28 3831-92

[5] Ogo Y, Hiramatsu H, Nomura K, Yanagi H, Kamiya T, Hirano M and Hosono H 2008 p -channel thin-film transistor using p-type oxide semiconductor, SnO Appl. Phys. Lett. 93032113

[6] Caraveo-Frescas J A, Nayak P K, Al-Jawhari H A, Granato D B, Schwingenschlogl U and Alshareef H N 2013 Record Mobility in Transparent p-Type Tin Monoxide Films and Devices by PhasenEngineering ACS Nano 7 5160-7

[7] Luo H, Liang L, Cao H, Dai M, Lu Y and Wang M 2015 Control of Ambipolar Transport in SnO Thin-Film Transistors by Back-Channel Surface Passivation for High Performance Complementary-like Inverters ACS Appl. Mater. Interfaces 7 $17023-31$

[8] Zhong C W, Lin H C, Tsai J R, Liu K C and Huang T Y 2016 Impact of gate dielectrics and oxygen annealing on tinoxide thin-film transistors Jpn. J. Appl. Phys. 55 04EG02

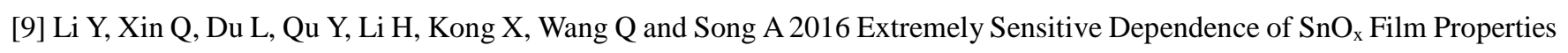
on Sputtering Power Sci. Rep. 636183

[10] Kim S H, Baek I H, Kim D H, Pyeon J J, Chung T M, Baek S H, Kim J S, Han J H and Kim S K 2017 Fabrication of high-performance p-type thin film transistors using atomic-layer-deposited SnO films J. Mater. Chem. C 5 3139-45 
[11] Fortunato E, Barros R, Barquinha P, Figueiredo V, Park S H K, Hwang C S, Martins R 2010 Transparent p-Type SnO Thin Film Transistors Produced by Reactive rf Magnetron Sputtering Followed by Low Temperature Annealing Appl. Phys. Lett. 97052105

[12] Togo A, Oba F, Tanaka I and Tatsumi K 2006 First-principles calculations of native defects in tin monoxide Phys. Rev. B 74195128

[13] Ogo Y, Hiramatsu H, Nomura K, Yanagi H, Kamiya T, Kimura M, Hirano M and Hosono H 2009 Tin monoxide as an s-orbital-based p-type oxide semiconductor: Electronic structures and TFT application Phys. Status Solidi A 206 21872091

[14] Nomura K, Kamiya T and Hosono H 2011 Ambipolar Oxide Thin-Film Transistor Adv. Mater. 23 3431-4

[15] Liang L and Cao H 2012 Ambipolar SnO thin-film transistors and inverters ECS Trans. 50 289-97

[16] Luo H, Liang L Y, Liu Q and Cao H T 2014 Magnetron-Sputtered SnO Thin Films for p-Type and Ambipolar TFT Applications ECS J. Solid State Sci. Technol. 3 Q3091-4

[17] Li D, Borkent E J, Nortrup R, Moon H, Katz H and Bao Z 2005 Humidity effect on electrical performance of organic thinfilm transistors Appl. Phys. Lett. 86042105

[18] Han Y J, Chio Y J, Jeong C Y, Lee D, Song S H and Kwon H I 2015 Environment-Dependent Bias Stress Stability of P-Type SnO Thin-Film Transistors IEEE Electron Device Lett. 36 466-8

[19] Chen Y C, Chang T C, Li H W, Chen S C, Lu J, Chung W F, Tai Y H and Tseng T Y 2010 Bias-induced oxygen adsorption in zinc tin oxide thin film transistors under dynamic stress Appl. Phys. Lett. 96262104

[21] Han Y J, Chio Y J, Cho I T, Jin S H, Lee J H and Kwon H I 2014 Improvement of Long-Term Durability and Bias Stress Stability in p-Type SnO Thin-Film Transistors Using a SU-8 Passivation Layer IEEE Electron Device Lett. 35 $1260-2$

[22] Park J S, Jeong J K, Chung H J, Mo Y G and Kim H D 2008 Electronic transport properties of amorphous indium-galliumzinc oxide semiconductor upon exposure to water Appl. Phys. Lett. 92072104

[23] Jeong J H, Yang H W, Park J S, Jeong J K, Mo Y G, Kim H D, Song J and Hwang C S 2008 Origin of Subthreshold Swing Improvement in Amorphous Indium Gallium Zinc Oxide Transistors Electrochem. and Solid-State Lett. 11 H1579

[24] Erslev P T, Sundholm E S, Presley R E, Hong D, Wager J F and Cohen J D 2009 Mapping out the distribution of electronic states in the mobility gap of amorphous zinc tin oxide Appl. Phys. Lett. 95192115

[25] Zhang B, Li H, Zhang X, Luo Y, Wang Q and Song A 2015 Performance regeneration of InGaZnO transistors with ultra- 
thin channels Appl. Phys. Lett. 106093506

[26] Hsieh H H, Kamiya T, Nomura K, Hosono H and Wu C C 2008 Modeling of amorphous $\mathrm{InGaZnO}_{4}$ thin film transistors and their subgap density of states Appl. Phys. Lett. 92133503 